\title{
Synthesis and Spectral Analysis of Pyridine Derivates
}

\author{
Aleksandra Wojciechowska ${ }^{*}$, Karolina Wieszczycka ${ }^{1}$, Grzegorz Framski ${ }^{2}$ \\ ${ }^{1}$ Poznan University of Technology, Institute of Chemical Technology and Engineering, Berdychowo St. 4, \\ 60-965 Poznan, Poland \\ ${ }^{2}$ Institute of Bioorganic Chemistry Polish Academy of Science, Noskowskiego \\ St. 12,14, 61-704 Poznan, Poland \\ Email: aleksandra.w.wojciechowska@doctorate.put.poznan.pl
}

\begin{abstract}
The present study described a detailed investigation of the synthesis of novel quaternary pyridinium salts: derivates of 1-(3-pyridine)undecane-1-one (1-propyl-3-undecanoylpyridinium bromide and 1-propyl-3-undecanoylpyridinium chloride) and $N$-decyloxypyridine-3-carboximidamide (3-[1-(N-decyloxyimine)ethyl]-1-propylpyridinium chloride and 3-[1-(N-decyloxyimine)ethyl]-1propylpyridinium bromide). The structures of the products were confirmed by spectral $\left({ }^{1} \mathrm{H}-\mathrm{NMR},{ }^{13} \mathrm{C}-\right.$ NMR, FT-IR) analysis, mass spectrometry methods. Estimation of the pharmacotherapeutic potential has been accomplished for synthesized compounds on the basis of Prediction of Activity Spectra for Substances (PASS programe).
\end{abstract}

Keywords: Quaternary pyridinium salts, synthesis, quarterisation reaction, $O$-alkylation, prediction of activity

\section{Introduction}

First reports about quaternary ammonium salts appeared in 1890, when Menchutkin first time conducted reaction of a tertiary amine with an alkyl halide [1]. The Menchutkin reactions (MR) with the exception of a few cases, are of the $\mathrm{S}_{\mathrm{N}} 2$ type. Primary halides are generally more reactive than secondary or tertiary halides [2], reactivity increase in the order: iodide $>$ bromide $>$ chloride [3-5]. The MR needs an appropriate polar solvent (such as alcohol, aceton etc.). In a strongly polar medium the reaction takes place more easily through the stabilization of the transition state and the charged products, which are more polar than the reactants.

From that time many studies of quaternary ammonium salts, were carried out what brought huge knowledge about their properties and utility. Those properties are used in many areas of our daily lives [6]. They are used as catalysts, substrates for ionic liquids synthesis, in textile industry or even as wood impregnation agents [7-9]. Moreover they may be used in cosmetics industries [10,11], nanomaterials synthesis or pharmaceuticals $[12,13]$. Besides they have biological active properties, which is the reason why they are very often used as bactericidals, fungicidals [13] and also as herbicides [6,14-19]. Pyridine oximes and their quaternary salts are also used as selective and efficient extractants in metals recovery from aqueous solution [20,21].

Therefore, the goal of the research was to synthesize novel quaternary pyridinium salts: derivates of 1(3-pyridine) undecane-1-one and $N$-decyloxypyridine-3-carboximidamide in large scale and with high yields. In this work, two starting compounds were proposed: the two-step synthesis of quaternary salts of 1-(3-pyridine)undecane-1-one (Scheme 1.) and the three-step synthesis of quaternary salts of $N$ decyloxypyridine-3-carboximidamide (Scheme 2). Both of these methods can be also used to synthesize substituent at position 2 and 4 of the pyridine ring. 


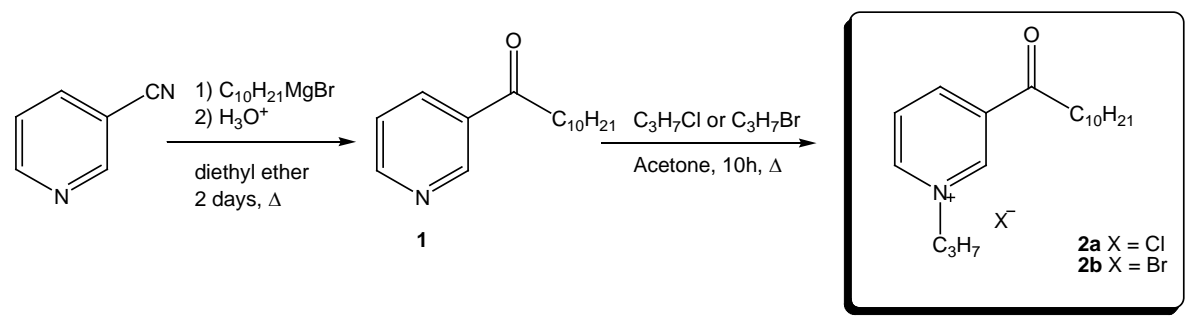

Scheme 1. Two-step synthesis of 1-propyl-3-undecanoylpyridinium bromide and 1-propyl-3-undecanoylpyridinium chloride (2a-b).



Scheme 2. Three-step synthesis of 3-[1-( $N$-decyloxyimine $)$ ethyl]-1-propylpyridinium chloride and $3-[1-(N$ decyloxyimine)ethyl]-1-propylpyridinium bromide (5a-b)

\section{$2 \quad$ Results and Discussion}

1-propyl-3-undecanoylpyridinium chloride (2a) and 1-propyl-3-undecanoylpyridinium chloride (2b) were synthesized using the two-step synthesis. In the first case, using the two-step synthesis, only derivatives of the 2-position of the pyridine ring were synthesized. In the first step mixture was heated under reflux and under anhydrous conditions for seven hours (solution is decolorized, it means the start of reaction). In the next step, during the addition of 3-cyanopyridine solution color changes from green to orange. After that, solution was neutralized by adding stoichiometric amount of aqueous solution of saturated ammonium chloride hydrochloric acid and sodium hydroxide until $\mathrm{pH}$ reached neutral. The resulting two layers can be easily separated and purified using chloroform, washed with brine and dried. The crude product obtained was chromatographed on a column of silica gel. The second step was a substitution reaction $\left(\mathrm{S}_{N} 2\right)$ with an appropriate halide in anhydrous acetone. The results of the reaction were summarized in Table 1.

Table 1. Yields and forms of synthesized derivates of of 1-(3-pyridine) undecane-1-one

\begin{tabular}{ccc}
\hline No & Yield [\%] & Form \\
\hline $\mathbf{1}$ & 89.5 & solid $\left(43.0-43.2^{\circ} \mathrm{C}\right)$ \\
$\mathbf{2 a}$ & 83.2 & solid $\left(40.3-41.0^{\circ} \mathrm{C}\right)$ \\
$\mathbf{2 b}$ & 92.4 & solid $\left(39.0-40.0^{\circ} \mathrm{C}\right)$ \\
\hline
\end{tabular}


Quaternary salts derivates of $N$-decyloxypyridine-3-carboximidamide were obtained by the oximation reaction of the 3-acetylpyridine with hydroxylamine hydrochloride in the presence of $\mathrm{NaOH}($ at $\mathrm{pH}=7$; ethanol-water mixture 4:1 (v/v)). The second step was the reaction of quaternization of obtained oxime. The last step was a quaternization reaction with an appropriate halide in anhydrous acetone. Products form and yields were summarized in Table 2.

Table 2. Yields and characteristic spectral data of synthesized derivates of $N$-decyloxypyridine-3-carboximidamide

\begin{tabular}{ccc}
\hline No & Yield [\%] & Form \\
\hline $\mathbf{3}$ & 97 & solid $\left(103^{\circ} \mathrm{C}\right)$ \\
$\mathbf{4}$ & 98 & oil \\
$\mathbf{5 a}$ & 92 & oil \\
$\mathbf{5 b}$ & 95 & oil \\
\hline
\end{tabular}

The potential pharmacological activities of the synthesized compounds have been also studied. A computer-aided drug discovery approach with the in silico Prediction of Activity Spectra for Substances (PASSs) program was used. The PASS software is useful for the study of biological activity of organic compounds. A few types of activities were predicted for a potential compound with the highest probability. If predicted activity $(\mathrm{PA})>0.8$, the substance is very likely to exhibit experimental activity and the chance of the substance being the analogue of a known pharmaceutical agent is also high. According to data from PASS program the most frequently predicted types of biological activity are: carboxypeptidase Taq inhibitor and pullulanase inhibitor.

Table 3. Probability "to be Active" (PA) values for predicted biological activity of compounds 1, 2a-b.

\begin{tabular}{llll}
\hline \multirow{2}{*}{ Focal predicted activity $(\mathbf{P A}>\mathbf{0 . 8 0})$} & \multicolumn{3}{c}{ Compound } \\
\cline { 2 - 4 } & $\mathbf{1}$ & $\mathbf{2 a}$ & $\mathbf{2 b}$ \\
\hline Carboxypeptidase Taq inhibitor & 0.898 & 0.853 & 0.870 \\
Pullulanase inhibitor & 0.875 & 0.808 & 0.878 \\
CYP2F1 substrate & 0.873 & - & - \\
Taurine dehydrogenase inhibitor & 0.873 & - & - \\
Pro-opiomelanocortin converting enzyme inhibitor & 0.856 & - & 0.809 \\
Superoxide dismutase inhibitor & 0.842 & - & - \\
Amine dehydrogenase inhibitor & 0.818 & - & - \\
Gluconate 5-dehydrogenase inhibitor & - & - & 0.817 \\
Maleate isomerase inhibitor & - & - & 0.801 \\
\hline
\end{tabular}

Table 4. Probability "to be Active" (PA) values for predicted biological activity of compounds 3,4 and 5a-b.

\begin{tabular}{lcccc}
\hline Focal predicted activity $($ PA $>$ 0.80) & \multicolumn{5}{c}{ Compound } \\
\cline { 2 - 5 } & $\mathbf{3}$ & $\mathbf{4}$ & $\mathbf{5 a}$ & $\mathbf{5 b}$ \\
\hline Muramoyltetrapeptide carboxypeptidase inhibitor & 0.892 & 0.846 & - & - \\
3-Hydroxybenzoate 4-monooxygenase inhibitor & 0.830 & - & - & - \\
Dehydro-L-gulonate decarboxylase inhibitor & 0.832 & - & - & - \\
Fragilysin inhibitor & 0.828 & - & - & - \\
Nicotinamidase inhibitor & 0.810 & - & - & - \\
Carboxypeptidase Taq inhibitor & 0.808 & 0.849 & 0.836 & 0.855 \\
Phobic disorders treatment & - & 0.947 & - & 0.887 \\
Sugar-phosphatase inhibitor & - & 0.882 & - & - \\
Pullulanase inhibitor & - & 0.832 & 0.872 & 0.839 \\
Gluconate 5-dehydrogenase inhibitor & - & 0.829 & - & - \\
\hline
\end{tabular}




\section{$3 \quad$ Experimental}

\subsection{Material and Methods}

Melting points were determined using a Boetius hot stage apparatus. The nuclear magnetic resonance (NMR) spectra were measured with a Bruker Avance II $400 \mathrm{MHz}$ UltraShield Plus spectrometer, operating at 400.6 and $101.2 \mathrm{MHz}$ for ${ }^{1} \mathrm{H}$ and ${ }^{13} \mathrm{C}$, respectively. The number of scans varied from 1000 to 5,000 per spectrum. The ${ }^{13} \mathrm{C}$ and ${ }^{1} \mathrm{H}$ chemical shifts were measured in $\mathrm{CDCl}_{3}$ or DMSO-d6 relative to an internal standard of TMS. Infrared spectra were recorded in the KBr pellets or solutions in chloroform using Vertex 70, Bruker Optics spectrophotometer. The ESI (electron spray ionization) mass spectra were recorded on API 4000 QTRAP, AB Sciex (Foster City, CA, USA) spectrometer. The sample solutions were prepared in methanol at the concentration of approximately $10^{-5} \mathrm{M}$. The standard ESIMS mass spectra were recorded at the cone voltage $30 \mathrm{~V}$. The low- and high-resolution mass spectra were recorded on an Intectra Mass AMD 402 (ionization method EI, $70 \mathrm{eV}$ ) spectrometer. Reaction progress and purity of the compounds were monitored by thin-layer chromatography(TLC) using precoated aluminum-backed silica plates (E. Merck, DC-60F 254 ) and monitored by UV lamp (UV 254 nm). Silica gel 60 (E. Merck 70-230 mesh) was used for column chromatography.

\subsection{Typical Procedure for the Preparation of Quaternary Salts of 1-(3-Pyridine) Undecane-1-One by Two-Step Method}

\subsubsection{Synthesis of 1-(3-pyridine)undecane-1-one (1)}

In round bottom flask, $0.2 \mathrm{~mol}(4.86 \mathrm{~g})$ dried magnesium $(\mathrm{POCH})$ and a crystal of iodine was placed. Then solution of $0.25 \mathrm{~mol}(42.75 \mathrm{~g}$ ) of decyl bromide (Sigma Aldrich) in $300 \mathrm{ml}$ dry diethyl ether $(\mathrm{POCH})$ was slowly added. The mixture was stirred under reflux for six hours. After that $0.2 \mathrm{~mol}$ pyridine-3-carbonitrile (Sigma Aldrich) was added and heated under reflux for 7 hours. The solution was neutralized by adding stoichiometric amount of aqueous solution of saturated ammonium chloride(POCH), next $15 \%$ hydrochloric acid $(15 \%, \mathrm{POCH})$ and sodium hydroxide $(20 \%, \mathrm{POCH})$. Then the resulting aqueous solution was extracted using chloroform, washed with brine and dried with anhydrous $\mathrm{MgSO}_{4}$. The crude product was chromatographed on silica gel $(200 \mathrm{~g})$ with toluenechloroform 5:1 (v/v) as an eluent.

1-(3-pyridine) undecane-1-one (1) $\mathrm{Rf}=0.61$ (chloroform-heptane 1:1).

3.2.2 Synthesis of 1-propyl-3-undecanoylpyridinium bromide and chloride (2a-b)

In round bottom flask, $0.1 \mathrm{~mol}$ (13.62 g) of 1-(3-pyridine)undecane-1-one and $0.125 \mathrm{~mol}$ propyl bromide or chloride $(15.25 \mathrm{~g} / 9.82 \mathrm{~g}$ ) (Sigma Aldrich) was dissolved in $100 \mathrm{ml}$ of dry acetone and warm up to $40^{\circ} \mathrm{C}$. Then mixture was filtered and concentrated under rotary evaporator. The crude product was recrystallized from acetone.

1-propyl-3-undecanoylpyridinium bromide (2a) $\mathrm{Rf}=0.46$ (chloroform-heptane 1:1)

1-propyl-3-undecanoylpyridinium chloride (2b) $\mathrm{Rf}=0.42$ (chloroform-heptane 1:1)

\subsection{Typical procedure for the preparation of quaternary salts of $\mathrm{N}$-decyloxypyridine-3- carboximidamide by three-step method}

\subsubsection{Synthesis of 1-(3-pyridine)ethane-1-one oxime (3)}

In round bottom flask, $0.25 \mathrm{~mol}$ of hydroxylamine hydrochloride $(17.4 \mathrm{~g})(\mathrm{POCH})$ and $0.2 \mathrm{~mol}$ sodium hydroxide $(8.0 \mathrm{~g})(\mathrm{POCH})$ was dissolved in $300 \mathrm{ml}$ of an ethanol-water mixture 4:1 (v/v) and warm up to $50^{\circ} \mathrm{C}$, after that 3-acetylpyridine (Sigma Aldrich) was added to the reaction mixture and heated under reflux by $4 \mathrm{~h}$. After that, hydrochloric acid or dry ice was added, to precipitate the product. The white powder was recrystallized from water. The spectroscopic parameters of 1-(3-pyridine)ethane-1-one oxime (FT-IR, ${ }^{1} \mathrm{H}-\mathrm{NMR}$, and ${ }^{13} \mathrm{C}-\mathrm{NMR}$ ) were identical to those given in the literature. Mp. $103^{\circ} \mathrm{C}$.

1-(3-pyridine)ethane-1-one oxime (3) $\mathrm{Rf}=0.54$ (chloroform: heptane 1:1).

3.3.2 Synthesis of $\mathrm{N}$-decyloxypyridine-3-carboximidamide (4)

In round bottom flask, $0.25 \mathrm{~mol}$ of 1-(3-pyridine)ethane-1-one oxime $(17.4 \mathrm{~g})$ and $0.2 \mathrm{~mol}$ sodium hydroxide $(8.0 \mathrm{~g})(\mathrm{POCH})$ was dissolved in $300 \mathrm{ml}$ of an isopropyl-water mixture 5:1 (v/v) and warm up 
to $50^{\circ} \mathrm{C}$, after that decyl bromide (Sigma Aldrich) was added to the reaction mixture and heated under reflux by $6 \mathrm{~h}$. After that, hydrochloric acid or dry ice was added, to precipitate the product. The crude product was chromatographed on silica gel (200 g) with toluene-chloroform 6:1 (v/v) as an eluent.

$N$-decyloxypyridine-3-carboximidamide (4) $\mathrm{Rf}=0.63$ (toluene-chloroform 1:1).

\subsubsection{Synthesis of 3-[1-( $N$-decyloxyimine)ethyl]-1-propylpyridinium chloride and} bromide(5a-b)

In round bottom flask, $0.1 \mathrm{~mol}(27.64 \mathrm{~g})$ of $N$-decyloxypyridine-3-carboximidamide and $0.125 \mathrm{~mol}$ propyl bromide or chloride (15.25 g/ $9.82 \mathrm{~g}$ ) (Sigma Aldrich) was dissolved in $100 \mathrm{ml}$ of dry acetone and warm up to $40^{\circ} \mathrm{C}$. Then mixture was filtered and concentrated under rotary evaporator. The crude product was recrystallized from acetone.

3-[1-(N-decyloxyimine)ethyl]-1-propylpyridinium chloride (5a) $\mathrm{Rf}=0.52$ (toluene-chloroform 1:1).

3-[1-(N-decyloxyimine)ethyl]-1-propylpyridinium bromide (5b) $\mathrm{Rf}=0.50$ (toluene-chloroform 1:1).

1-(3-pyridine) undecane-1-one (1)- FT-IR $\left[\mathrm{cm}^{-1}\right]: 2890 ; 1690 ; 1730 ; 1450 ;{ }^{1} \mathrm{H} \mathrm{NMR}\left(\mathrm{CDCl}_{3}, \mathrm{DMSO}\right) \delta$ in ppm: $9.15(\mathrm{Py}-2,1 \mathrm{H}, \mathrm{s}) ; 8.75\left(\mathrm{Py}-6,1 \mathrm{H}, \mathrm{dd}, \mathrm{J}_{1}=3.17 \mathrm{~Hz}, \mathrm{~J}_{2}=6.36 \mathrm{~Hz}\right) ; 8,23\left(\mathrm{Py}-4,1 \mathrm{H}, \mathrm{dd}, \mathrm{J}_{1}=3.18 \mathrm{~Hz}\right.$, $\left.\mathrm{J}_{2}=12.47 \mathrm{~Hz}\right) ; 7.4(\mathrm{Py}-5,1 \mathrm{H}, \mathrm{t}) ; 2.96(2 \mathrm{H}, \mathrm{t}) ; 1.73(2 \mathrm{H}, \mathrm{qw}) ; 1.37-1.24(14 \mathrm{H}, \mathrm{m}) ; 0.86(3 \mathrm{H}, \mathrm{t}) ;{ }^{13} \mathrm{C} \mathrm{NMR}$ $\left(\mathrm{CDCl}_{3},\right)$ $\delta$ in ppm: $198.7(\mathrm{C}=\mathrm{O}) ; 152.8(\mathrm{Py}-2) ; 149.1$ (Py-6); 134.8 (Py-4); 131.7 (Py-3); 123.0 (Py-5); $39.1 ; 38.4 ; 37.4 ; 35.2 ; 31.3 ; 29.0 ; 28.8 ; 23.5 ; 22.1 ; 13.6 ;\left(\right.$ ESI-MS) m/z: $247.38(\mathrm{M}+\mathrm{H})^{+}$; HRMS (EI): Calcd for $\mathrm{C}_{16} \mathrm{H}_{25} \mathrm{NO}(\mathrm{M})^{+}: \mathrm{m} / \mathrm{z}$ 247.1936. Found: $\mathrm{m} / \mathrm{z} 247.1935$. Rf=0.61 (chloroform-heptane 1:1).

1-propyl-3-undecanoylpyridinium bromide (2a)- FT-IR $\left[\mathrm{cm}^{-1}\right]: 2890 ; 1680 ; 1735 ; 1450 ; 860 ;{ }^{1} \mathrm{H}$ NMR $\left(\mathrm{CDCl}_{3}, \mathrm{DMSO}\right) \delta$ in ppm: $9.20(\mathrm{Py}-2,1 \mathrm{H}, \mathrm{s}) ; 8.60\left(\mathrm{Py}-6,1 \mathrm{H}, \mathrm{dd}, \mathrm{J}_{1}=3.16 \mathrm{~Hz}, \mathrm{~J}_{2}=6.34 \mathrm{~Hz}\right) ; 8,20(\mathrm{Py}-4$, $\left.1 \mathrm{H}, \mathrm{dd}, \mathrm{J}_{1}=3.16 \mathrm{~Hz}, \mathrm{~J}_{2}=12.30 \mathrm{~Hz}\right) ; 7.45(\mathrm{Py}-5,1 \mathrm{H}, \mathrm{t}) ; 3.66\left(\mathrm{~N}_{-} \mathrm{CH}_{2} ; 2 \mathrm{H}, \mathrm{t}\right) ; 2.96(2 \mathrm{H}, \mathrm{t}) ; 1.75(2 \mathrm{H}, \mathrm{qw})$; 1.36-1.23 (18H, m); $0.83(6 \mathrm{H}, \mathrm{t}) ;{ }^{13} \mathrm{C} \mathrm{NMR}\left(\mathrm{CDCl}_{3},\right) \delta$ in ppm: $200.1(\mathrm{C}=\mathrm{O}) ; 148.1(\mathrm{Py}-2) ; 146.0(\mathrm{Py}-6)$; 145.6 (Py-4); 141.2 (Рy-3); 137.6 (Py-5); 64.3; 38.4; 29.6; 29.4; 29.0; 25.6; 22.7; 14.1; 12.5;(ESI-MS) m/z: $370.37(\mathrm{M}+\mathrm{H})^{+}$; HRMS (EI): Calcd for $\mathrm{C}_{19} \mathrm{H}_{32} \mathrm{BrNO}(\mathrm{M})^{+}: \mathrm{m} / \mathrm{z}$ 369.1167. Found: $\mathrm{m} / \mathrm{z}$ 369.1168. $\mathrm{Rf}=0.46$ (chloroform-heptane 1:1).

1-propyl-3-undecanoylpyridinium chloride (2b)- FT-IR $\left[\mathrm{cm}^{-1}\right]: 2890 ; 1725 ; 1680 ; 1450 ; 770 ;{ }^{1} \mathrm{H}$ NMR $\left(\mathrm{CDCl}_{3}, \mathrm{DMSO}\right) \delta$ in ppm: $9.22(\mathrm{Py}-2,1 \mathrm{H}, \mathrm{s}) ; 8.70\left(\mathrm{Py}-6,1 \mathrm{H}, \mathrm{dd}, \mathrm{J}_{1}=3.16 \mathrm{~Hz}, \mathrm{~J}_{2}=6.24 \mathrm{~Hz}\right) ; 8,30(\mathrm{Py}-4$, $\left.1 \mathrm{H}, \mathrm{dd}, \mathrm{J}_{1}=3.16 \mathrm{~Hz}, \mathrm{~J}_{2}=12.50 \mathrm{~Hz}\right) ; 7.45(\mathrm{Py}-5,1 \mathrm{H}, \mathrm{t}) ; 3.50\left(\mathrm{~N}_{-} \mathrm{CH}_{2} ; 2 \mathrm{H}, \mathrm{t}\right) ; 2.96(2 \mathrm{H}, \mathrm{t}) ; 1.73(2 \mathrm{H}, \mathrm{qw})$; 1.36-1.24 (18H, m); $0.86(6 \mathrm{H}, \mathrm{t}) ;{ }^{13} \mathrm{C} \mathrm{NMR}\left(\mathrm{CDCl}_{3},\right) \delta$ in ppm: $200.1(\mathrm{C}=\mathrm{O}) ; 148.1$ (Py-2); 145.6 (Py-6); 137.6 (Рy-4); 132.3 (Рy-3); 128.4 (Рy-5); 64.3; 38.4; 31.9; 29.7; 29.4; 29.2; 25.6; 22.8; 14.1; 12.5; (ESI-MS) $\mathrm{m} / \mathrm{z}: 325.92(\mathrm{M}+\mathrm{H})^{+}$; HRMS (EI): Calcd for $\mathrm{C}_{19} \mathrm{H}_{32} \mathrm{ClNO}(\mathrm{M})^{+}: \mathrm{m} / \mathrm{z} 325.2172$. Found: $\mathrm{m} / \mathrm{z} 325.2175$. $\mathrm{Rf}=0.42$ (chloroform-heptane $1: 1$ ).

1-(3-pyridine)ethane-1-one oxime (3) - FT-IR $\left[\mathrm{cm}^{-1}\right]: 3600 ; 2965 ; 1686 ; 1435 ; 1100 ;{ }^{1} \mathrm{H}$ NMR $\left(\mathrm{CDCl}_{3}, \mathrm{DMSO}\right) \delta$ in ppm: $9.10(\mathrm{O}-\mathrm{H}, 1 \mathrm{H}, \mathrm{s}) ; 8,84(\mathrm{Py}-2,1 \mathrm{H}, \mathrm{s}) ; 8.50(\mathrm{Py}-6,1 \mathrm{H}, \mathrm{d}) ; 7.82(\mathrm{Py}-5,1 \mathrm{H}, \mathrm{t})$; 7.22 (Py-4, 1H, d); $0.84(3 \mathrm{H}, \mathrm{t}) ;{ }^{13} \mathrm{C} \mathrm{NMR}\left(\mathrm{CDCl}_{3}\right.$ ) $\delta$ in ppm: 150.0 (Py-2); 148.2 (Py-6); 147.5 (Py-4); $133.2(\mathrm{Py}-3) ; 132.4(\mathrm{Py}-5) ; 121.6(\mathrm{C}=\mathrm{N}) ; 13.7 ;{ }^{13} \mathrm{C} \mathrm{NMR}\left(\mathrm{CDCl}_{3},\right) \delta$ in ppm: (ESI-MS) $\mathrm{m} / \mathrm{z}: 135.16$ $(\mathrm{M}+\mathrm{H})^{+}$; $\operatorname{HRMS}(\mathrm{EI})$ : Calcd for $\mathrm{C}_{7} \mathrm{H}_{8} \mathrm{~N}_{2} \mathrm{O}(\mathrm{M})^{+}: \mathrm{m} / \mathrm{z}$ 136.0637. Found: $\mathrm{m} / \mathrm{z}$ 136.0636. Rf $=0.54$ (chloroform: heptane 1:1).

$N$-decyloxypyridine-3-carboximidamide (4) - FT-IR $\left[\mathrm{cm}^{-1}\right]: 3200 ; 2986 ; 1678 ; 1450 ; 1020 ;{ }^{1} \mathrm{H}$ NMR $\left(\mathrm{CDCl}_{3}, \mathrm{DMSO}\right) \delta$ in ppm: 8,85 (Py-2, 1H, s); $8.55(\mathrm{Py}-6,1 \mathrm{H}, \mathrm{d}) ; 7.94(\mathrm{Py}-5,1 \mathrm{H}, \mathrm{t}) ; 7.23(\mathrm{Py}-4,1 \mathrm{H}, \mathrm{d})$; $4.18\left(\mathrm{O}-\mathrm{CH}_{2} ; 2 \mathrm{H}, \mathrm{t}\right) ; 2.21(3 \mathrm{H}, \mathrm{s}) ; 1.87(2 \mathrm{H}, \mathrm{m}) ; 1.25(14 \mathrm{H}, \mathrm{m}) ; 0.85(3 \mathrm{H}, \mathrm{t}) ;{ }^{13} \mathrm{C} \mathrm{NMR}\left(\mathrm{CDCl}_{3},\right) \delta$ in ppm: 150.1 (Py-2); $149.2(\mathrm{Py}-6) ; 146.8(\mathrm{Py}-4) ; 132.6(\mathrm{Py}-3) ; 132.0(\mathrm{Py}-5) ; 121.6(\mathrm{C}=\mathrm{N}) ; 74.10 ; 31.4 ; 29.6 ; 29.0 ;$ 28.8; 27.4; 25.5; 25.2; 22.1; 13.7; 11.7; (ESI-MS) m/z: $276.42(\mathrm{M}+\mathrm{H})^{+}$; HRMS (EI): Calcd for $\mathrm{C}_{17} \mathrm{H}_{28} \mathrm{~N}_{2} \mathrm{O}$ $(\mathrm{M})^{+}: \mathrm{m} / \mathrm{z}$ 276.2202. Found: $\mathrm{m} / \mathrm{z} 276.2204 . \mathrm{Rf}=0.63$ (toluene-chloroform 1:1).

3-[1-(N-decyloxyimine)ethyl]-1-propylpyridinium chloride (5a) - FT-IR [cm $\left.{ }^{-1}\right]: 3100 ; 2985 ; 1650 ; 1465$; 1000; 760; ${ }^{1} \mathrm{H}$ NMR $\left(\mathrm{CDCl}_{3}\right.$,DMSO) $\delta$ in ppm: 8,80 (Py-2, 1H, s); 8.53 (Py-6, 1H, d); 7.90 (Py-5, 1H, t);

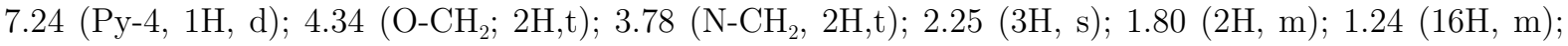
$0.86(6 \mathrm{H}, \mathrm{t}) ;{ }^{13} \mathrm{C}$ NMR $\left(\mathrm{CDCl}_{3},\right) \delta$ in ppm: $150.5(\mathrm{Py}-2) ; 148.2(\mathrm{Py}-6) ; 147.8(\mathrm{Py}-4) ; 135.6(\mathrm{Py}-3) ; 132.5$ $(\mathrm{Py}-5) ; 121.8(\mathrm{C}=\mathrm{N}) ; 74.10 ; 64.78 ; 31.4 ; 30.8 ; 29.6 ; 29.4 ; 29.0 ; 28.8 ; 27.4 ; 25.5 ; 25.2 ; 22.1 ; 13.7 ; 11.7 ;{ }^{13} \mathrm{C}$ NMR $\left(\mathrm{CDCl}_{3},\right) \delta$ in ppm: (ESI-MS) m/z: $354.96(\mathrm{M}+\mathrm{H})^{+}$; HRMS (EI): Calcd for $\mathrm{C}_{20} \mathrm{H}_{35} \mathrm{ClN}_{2} \mathrm{O}(\mathrm{M})^{+}$: $\mathrm{m} / \mathrm{z}$ 354.2438. Found: $\mathrm{m} / \mathrm{z}$ 354.2441. $\mathrm{Rf}=0.52$ (toluene-chloroform 1:1).

3-[1-(N-decyloxyimine)ethyl]-1-propylpyridinium bromide (5b) - FT-IR [cm $\left.{ }^{-1}\right]: 3200 ; 2984 ; 1670 ; 1540 ;$ 1460; 1010; 880; ${ }^{1} \mathrm{H}$ NMR ( $\left.\mathrm{CDCl}_{3}, \mathrm{DMSO}\right) \delta$ in ppm: 8,85 (Py-2, 1H, s); 8.55 (Py-6, 1H, d); 7.95 (Py-5, $1 \mathrm{H}, \mathrm{t}) ; 7.22(\mathrm{Py}-4,1 \mathrm{H}, \mathrm{d}) ; 4.44\left(\mathrm{O}_{-} \mathrm{CH}_{2} ; 2 \mathrm{H}, \mathrm{t}\right) ; 3.83\left(\mathrm{~N}_{-} \mathrm{CH}_{2}, 2 \mathrm{H}, \mathrm{t}\right) ; 2.25(3 \mathrm{H}, \mathrm{s}) ; 1.85(2 \mathrm{H}, \mathrm{m}) ; 1.24(16 \mathrm{H}$, $\mathrm{m}) ; 0.85$ (6H, t); ${ }^{13} \mathrm{C}$ NMR $\left(\mathrm{CDCl}_{3},\right) \delta$ in ppm: $151.5(\mathrm{Py}-2) ; 148.4(\mathrm{Py}-6) ; 147.0(\mathrm{Py}-4) ; 135.4(\mathrm{Py}-3)$; 
$132.2(\mathrm{Py}-5) ; 121.7(\mathrm{C}=\mathrm{N}) ; 75.1 ; 64.7 ; 32.4 ; 30.9 ; 29.8 ; 29.6 ; 29.2 ; 28.8 ; 27.5 ; 25.4 ; 25.2 ; 22.1 ; 13.0 ; 11.2 ;$ (ESI-MS) m/z: $399.41(\mathrm{M}+\mathrm{H})^{+}$; HRMS (EI): Calcd for $\mathrm{C}_{20} \mathrm{H}_{35} \mathrm{BrN}_{2} \mathrm{O}(\mathrm{M})^{+}: \mathrm{m} / \mathrm{z}$ 398.1933. Found: $\mathrm{m} / \mathrm{z}$ 398.1931. $\mathrm{Rf}=0.50$ (toluene-chloroform 1:1).

\section{Conclusion}

The efficient two- and three-step procedure for new quaternary pyridinium salts (1-propyl-3undecanoylpyridinium bromide, 1-propyl-3-undecanoylpyridinium chloride, synthesis on a large scale were developed. These new compounds were characterized by spectroscopic methods and may find applications in pharmacology.

Acknowledgments: The study was financed within the Polish National Centre of Science funds according to decision no. DEC-2015/17/N/ST8/00285. We acknowledge the support of the Ministry of Science and Higher Education and European Fund for Regional Development under grant UDAPOIG.02.01.00-30-182/09 ( ${ }^{1} \mathrm{H}$ and ${ }^{13} \mathrm{C}$ NMR analysis).

\section{References}

1. N. Menschutkin, "Beiträgen zur kenntnis der affinitätskoeffizienten der alkylhaloide und der organischen amine", Zeitschrift für Physikalische Chemie, vol. 5, pp. 589-601, 1890.

2. C. R. Noller, R. Dinsmore, "The reaction of alkyl bromides with pyridine", Journal of the American Chemical Society, vol. 54, pp. 1025-1034, 1932.

3. H. T. Clarke, "The relation between reactivity and chemical constitution of certain halogen compounds", Journal of the Chemical Society, vol. 97. pp. 416-429, 1910.

4. C. A. Winkler, C. N. Hinschelwood, "The factors determining the velocity of reactions in solution. The formation of quaternary ammonium salts in benzene solution", Journal of the Chemical Society, pp. 1147-1151, 1935.

5. D. L. Comins, S. O'Connor, R. S. Al-awar, "Pyridines and their Benzo Derivatives: Reactivity at the Ring", Comprehensive Heterocyclic Chemistry III, Reference Module in Chemistry, Molecular Sciences and Chemical Engineering, vol.7, pp.41-99, 2008.

6. R. Mikláš, N. Miklášová, M. Bukovsky, B. Horváth, J. Kubincová , F. Devínsky, "Synthesis, surface and antimicrobial properties of some quaternary ammonium homochiral camphor sulfonamides", European Journal of Pharmaceutical Sciences, vol. 65, pp. 29-37, 2014.

7. P. Madaan P, V. K. Tyagi, "Quaternary pyridinium salts: a review", Journal of Oleo Science, vol. 57, no. 4, pp. 197-215, 2008.

8. Y. H. Kim, G. Sun, "Functional finishing of acrylic and cationic dyeable fabrics: intermolecular interactions", Textile Research Journal, vol. 72, pp. 1052-1056, 2002.

9. S. Shirakawa, K. Liu, K. Maruoka, "Catalytic asymmetric synthesis of axially chiral o-odoanilides by phasetransfer catalyzed alkylations", Journal of the American Chemical Society, vol. 134, no. 2, pp. 916-919, 2002.

10. M. Jadhav, R. S. Kalhapure, S. Rambharose, C. Mocktar, T. Govender, "Synthesis, characterization and antibacterial activity of novel heterocyclic quaternary ammonium surfactants", Journal of Industrial and Engineering Chemistry, article in press, 2016.

11. M. V. Pugachev, N. V. Shtyrlin, S. V. Sapozhnikov, L. P. Sysoeva, A. G. Iksanova, E. V. Nikitina, R. Z. Musin, O. A. Lodochnikova, E. A. Berdnikova, Y. G. Shtyrlin, "Bis-phosphonium salts of pyridoxine: The relationship between structure and antibacterial activity", Bioorganic \& Medicinal Chemistry, vol. 21, pp. 7330-7342, 2013.

12. B. Sarkar, Y. Xi, M. Megharaj, R. Naidu, "Orange II adsorption on palygorskites modified with alkyl trimethylammonium and dialkyl dimethylammonium bromide - an isothermal and kinetic study", Applied Clay Science, vol. 51, pp. 370-374, 2011.

13. B. Dmochowska, J. Piosik, A. Woziwodzka, K.Sikora, A. Wiśniewski, G. Węrzyn, "Mutagenicity of quaternary ammonium salts containing carbohydrate moieties", Journal of Hazardous Materials, vol. 193, pp. 272-278, 2011.

14. J. Krysiński, J. Placzek, A. Skrzypczak, B. Predki, „Analysis of relationships between structure, surface properties, and antimicrobial activity of quaternary ammonium chlorides", QSAR \& Combinatorial Science, vol. 28, pp. 995-1002, 2009.

15. E. Grabińska-Sota, "Genotoxicity and biodegradation of quaternary ammonium salts in aquatic environments", Journal of Hazardous Materials, vol. 195, pp. 182-187, 2011. 
16. E. Obłąk, A. Piecuch, A. Krasowska, J. Łuczyński, „Antifungal activity of gemini quaternary ammonium salts” Microbiological Research, vol.168, pp. 630-638, 2013.

17. E. Abele, R. Abele, E. Lukevics, "Pyridine oxime: synthesis, reactions and biological activity. Review", Chemistry of Heterocyclic Compounds, vol. 39, no. 7, pp. 825-865, 2003.

18. P. Madaan, V. K. Tyagi, "Quaternary Pyridinium Salts: A Review", Journal of Oleo Science, vol. 57, pp. 197215,2008

19. W. Sliwa, "Quaternary salts of pyridines and related compounds", Current Organic Chemistry, vol. 7, pp. 9951048,2003

20. K. Wieszczycka, A. Wojciechowska, M. Krupa, R. Kordala-Markiewicz, "Quaternary pyridinium ketoximes zinc extractants from chloride solutions", Journal Chemical Engineering Data, vol. 11, pp. 3207-3215, 2013.

21. K. Wejman-Gibas, K. Wieszczycka, A. Wojciechowska, K. Ochromowicz, P. Pohl, "Extraction of molybdenum(VI) from sulfate media by 3-pyridineketoxime and its quaternary salts", Separation and Purification Technology, vol. 158, pp. 71-79, 2016. 